\title{
A PRATICA BASEAdA EM EVIDÊNCIAS: CONSIDERAÇÕES TEÓRICAS PARA SUA IMPLEMENTAÇÃo NA ENFERMAGEM PERIOPERATÓRIA
}

Cristina Maria Galvão ${ }^{1}$ Namie Okino Sawada ${ }^{1}$

Lídia Aparecida Rossi ${ }^{2}$

Galvão CM, Sawada NO, Rossi LA. A prática baseada em evidências: considerações teóricas para sua implementação na enfermagem perioperatória. Rev Latino-am Enfermagem 2002 setembro-outubro; 10(5):690-5.

A prática baseada em evidências possibilita a melhoria da qualidade da assistência de enfermagem prestada ao cliente/paciente. O presente artigo tem como objetivo oferecer subsídios que proporcionem reflexões no cenário da enfermagem perioperatória. Assim, fundamentadas na literatura, tecemos considerações acerca da prática baseada em evidências; conceitos gerais, importância e barreiras para sua implementação na prática profissional com a finalidade de incentivar o enfermeiro cirúrgico a buscar conhecimento científico por meio do desenvolvimento de pesquisas e utilização de seus resultados.

DESCRITORES: enfermagem perioperatória, pesquisa, planejamento de assistência do paciente

\section{EVIDENGE-BASED PRACTICE: THEORETICAL CONSIDERATIONS ON ITS IMPLEMENTATION IN PERIOPERATIVE NURSING}

Evidence-based practice enables the quality improvement of the nursing care delivered to clients/patients. This work aimed at providing elements that will lead to reflections on the scenario concerning perioperative nursing. Accordingly, based on literature, authors have made considerations regarding evidence-based nursing practice, general concepts, its importance and the obstacles to its implementation in professional practice, aiming at encouraging surgical nurses to seek for scientific knowledge by developing research and applying its results.

DESCRIPTORS: perioperative nursing, research, patient's care planning

\section{LA PRACTICA BASAdA EN EVIDENCIAS: CONSIDERACIONES TEÓRICAS PARA SU IMPLEMENTACIÓN EN LA ENFERMERÍA PERIOPERATÓRIA}

La práctica basada en evidencias posibilita la mejora de la calidad de la atención de enfermería ofrecida al paciente/ cliente. Este artículo tiene como objetivo ofrecer subsídios para reflexiones en el escenario de la enfermería perioperatória. Así, fundamentadas en la literatura, las autoras elaboraron consideraciones sobre la práctica de enfermería basada en evidencias; conceptos generales, importancia y dificultades para su implementación en la práctica profesional con la finalidad de incentivar el enfermero quirúrgico a buscar conocimiento científico a través del desarrollo de investigaciones y utilización de sus resultados.

DESCRIPTORES: enfermería perioperatória, investigación, planeación de la atención al paciente

${ }^{1}$ Enfermeira, Professor Doutor, e-mail: crisgalv@eerp.usp.br; ${ }^{2}$ Enfermeira, Professor Associado. Escola de Enfermagem de Ribeirão Preto da Universidade de São Paulo, Centro Colaborador da OMS para o desenvolvimento da pesquisa em enfermagem 


\section{INTRODUÇÃO}

Em 1889, nos Estados Unidos da América (EUA), a enfermagem de sala de operação (SO) foi considerada uma área de especialização, tornando-se a primeira especialidade na enfermagem. Nas primeiras décadas do século XX, o enfermeiro de $S O$ era responsável pelo preparo do ambiente cirúrgico, auxílio da equipe médica e orientação de estudantes de enfermagem.

A Association of Operating Room Nurses (AORN) foi fundada em 1949, com os principais objetivos de formar um corpo de conhecimento para os enfermeiros de SO, promover ao paciente cirúrgico um ótimo cuidado por meio de programas educacionais e constituir uma associação para beneficiar todos profissionais que atuam nessa área. A partir da década de 60 , uma das metas assumidas pela AORN foi a melhoria da qualidade da assistência prestada ao paciente cirúrgico.

O início da prática de enfermagem em centro cirúrgico, no Brasil, ocorreu devido à ausência de pessoal capacitado para atender às necessidades da equipe médica, principalmente em relação ao preparo da sala de operação, artigos médico-hospitalares e equipamentos ${ }^{(1)}$.

Com o desenvolvimento da cirurgia, no final da década de 60 e início dos anos 70, surgiram os primeiros estudos orientados para a assistência de enfermagem prestada ao paciente na unidade de centro cirúrgico.

A enfermagem perioperatória "inclui os períodos pré-operatório, intra-operatório e pós-operatório da experiência cirúrgica do paciente"(2). O enfermeiro elabora o levantamento de dados sobre o paciente; coleta, organiza e prioriza os dados do paciente; estabelece o diagnóstico de enfermagem; desenvolve e implementa um plano de cuidados de enfermagem; e avalia aqueles cuidados em termos dos resultados alcançados pelo paciente ${ }^{(2)}$. Em outras palavras, o enfermeiro utiliza o processo de enfermagem como metodologia assistencial para o planejamento e implementação dos cuidados de enfermagem no período perioperatório.

Fundamentadas na nossa experiência profissional e na literatura, podemos afirmar que o procedimento anestésico cirúrgico é uma situação estressante tanto para o paciente como para as equipes médica e de enfermagem envolvidas.

O paciente, ao deparar-se com a cirurgia, é acometido de medos, tais como: medo da morte, da anestesia, do desconhecido, do câncer; além disso, apresenta preocupações em relação a problemas financeiros, responsabilidades familiares e compromissos no emprego.

Diante das características especificas do paciente cirúrgico, entendemos que a sistematização da assistência de enfermagem perioperatória possibilita a melhoria da qualidade da assistência prestada ao paciente, pois torna-se um processo individualizado, planejado, avaliado e, principalmente, contínuo, ou seja, abrange os períodos pré, intra e pós-operatório da experiência cirúrgica do paciente.

A visita pré-operatória de enfermagem ao paciente cirúrgico é o início da sistematização da assistência de enfermagem perioperatória. Esse procedimento é indispensável para o preparo físico e emocional do paciente ${ }^{(3)}$. Consiste em uma atividade do enfermeiro que possibilita uma interação efetiva, e esse profissional poderá detectar, solucionar e, quando necessário, encaminhar os problemas enfrentados pelo paciente.

A fase seguinte da sistematização da assistência de enfermagem perioperatória é a assistência ao paciente no período trans-operatório, ou seja, o período que compreende a recepção do paciente no centro cirúrgico, até o seu encaminhamento para a sala de recuperação pós-anestésica. O enfermeiro por meio de intervenções de enfermagem efetivas poderá minimizar os riscos decorrentes do procedimento anestésico cirúrgico.

A última fase da sistematização da assistência de enfermagem perioperatória é o período pós-operatório, e a equipe de enfermagem necessita estar preparada para possíveis complicações que possam ocorrer ao paciente nesse período. A complexidade de ações e a inter-relação das três fases da experiência cirúrgica do paciente, a nosso ver, justificam a importância da sistematização da assistência de enfermagem perioperatória e a utilização de conhecimento científico para esse embasamento.

Nesse cenário, entendemos que o processo de enfermagem pode ser empregado como metodologia assistencial pelo enfermeiro para o planejamento e implementação dos cuidados de enfermagem necessários ao paciente cirúrgico.

O processo de enfermagem é uma atividade deliberada, lógica e racional, por meio da qual a prática de enfermagem é desempenhada sistematicamente. Enquanto um método para organização da assistência, essa metodologia compreende cinco passos interrelacionados: coleta de dados, diagnóstico, planejamento, 
implementação e avaliação. Assim, o processo de enfermagem é um método sistemático e organizado para prestar cuidados de enfermagem individualizados, enfocando as respostas humanas de uma pessoa ou grupos, a problemas de saúde reais ou potenciais ${ }^{(4)}$. A realização de cada etapa dessa metodologia de assistência implica a prática baseada em evidências, tanto no que se refere aos dados coletados pelo enfermeiro (evidência baseada nas informações e nas condições clínicas do paciente) quanto ao julgamento clínico realizado por esse profissional para estabelecer os diagnósticos de enfermagem, planejar e avaliar as respostas à assistência implementada.

A prática baseada em evidências é um processo de descoberta, avaliação e aplicação de evidências científicas para o tratamento e gerenciamento da saúde ${ }^{(5)}$. É o cuidado guiado por meio de resultados de pesquisas, consenso de especialistas ou a combinação de ambos ${ }^{(6)}$.

Procurando oferecer subsídios que proporcionem reflexões no cenário da enfermagem perioperatória, apresentaremos, neste artigo, os conceitos gerais, importância e barreiras para a implementação da prática baseada em evidências.

\section{A PRÁTICA BASEADA EM EVIDÊNCIAS}

A prática baseada em evidências nasceu no Canadá e tem sido integrada no Sistema Nacional de Saúde do Reino Unido. A promoção da prática baseada em evidências no Reino Unido não ocorreu no vácuo, mas sim, como reflexo da necessidade de aumentar a eficiência e qualidade dos serviços de saúde, bem como diminuir os custos operacionais. Em 1991, o Departamento de Saúde, numa tentativa de remediar o déficit de conhecimento baseado em pesquisas, lançou um programa nacional com dois objetivos principais: o primeiro era assegurar que o cuidado prestado pelo Sistema Nacional de Saúde fosse baseado em pesquisas relevantes para melhorar a saúde da nação, e o segundo era que a utilização de pesquisas e o seu desenvolvimento deveria tornar-se parte integral dos serviços de saúde e que os administradores, equipe médica, de enfermagem e outros profissionais deveriam tomar decisões diárias baseadas nos resultados de investigações $^{(7)}$.

A origem da enfermagem baseada em evidências pode ser considerada no movimento da medicina baseada em evidências, a qual é definida como o consciencioso, explícito e criterioso uso da melhor evidência para tomar decisão sobre o cuidado individual do paciente. A prática da medicina baseada em evidências significa a integração da experiência clínica individual com a melhor evidência externa avaliada, oriunda de revisão sistemática de pesquisas $^{(8)}$.

A medicina baseada em evidências consiste em um novo paradigma, desenvolvido por estudiosos da Universidade McMaster (Canadá), na década de 80; "tratase de um processo seqüencial, constituído pelas seguintes etapas: $1^{a}$ - levantamento do problema e formulação da questão, $2^{a}$ - pesquisa da literatura correspondente; $3^{a}$ - avaliação e interpretação dos trabalhos coletados mediante critérios bem definidos; $4^{\mathrm{a}}$ - utilização das evidências encontradas, em termos assistenciais, de ensino e/ou de elaboração científica"(9).

A enfermagem baseada em evidências é definida como o uso consciencioso, explícito e criterioso de informações derivadas de teorias, pesquisas para a tomada de decisão sobre o cuidado prestado a indivíduos ou grupo de pacientes, levando em consideração as necessidades individuais e preferências ${ }^{(10)}$.

A prática baseada em evidências não conta com a intuição, observações não sistematizadas ou princípios patológicos. Ela enfatiza o uso de pesquisas para guiar a tomada de decisão clínica. Essa abordagem requer o aprendizado de novas habilidades para o uso de diferentes processos para a tomada de decisão. Essas habilidades incluem a aplicação formal das regras da evidência ao avaliar a literatura. Assim, a prática baseada em evidências combina a pesquisa com a experiência clínica e as preferências do paciente para realizar uma decisão sobre um problema específico ${ }^{(11)}$.

A evidência é caracterizada como alguma coisa que fornece provas para a tomada de decisão, abrange resultados de pesquisas, bem como consenso de especialistas reconhecidos; dentro de uma organização deve ser incluído fatos ou dados oriundos do trabalho desenvolvido ${ }^{(12)}$.

A força da evidência pode ser categorizada em cinco níveis, a saber: nível 1, evidência forte de, pelo menos, uma revisão sistemática de múltiplos estudos randomizados, controlados, bem delineados; nível 2, evidência forte de, pelo menos, um estudo randomizado, controlado, de delineamento apropriado e tamanho adequado; nível 3, evidência de estudos bem delineados 
sem randomização, grupo único pré e pós-coorte, séries temporais ou caso-controle pareado; nível 4, evidência de estudos bem delineados não experimentais, realizados em mais de um centro ou grupo de pesquisas; nível 5 , opiniões de autoridades respeitadas, baseadas em evidências clínicas, estudos descritivos ou relatórios de comitês de especialistas ${ }^{(13)}$.

A enfermagem, em comparação com a medicina, ainda não tem pesquisas suficientes para formar um corpo científico de conhecimento necessário para sustentar a prática baseada em evidências, principalmente estudos com evidências nível $1^{(12,14)}$. Em contrapartida, existe uma cultura que não reconhece a importância de pesquisas com abordagem qualitativa, a qual dificulta o incremento da enfermagem baseada em evidências ${ }^{(15)}$.

O desenvolvimento de pesquisas na enfermagem é fundamental, pois permite a construção de um corpo de conhecimento próprio, propicia a melhoria da assistência de enfermagem prestada ao cliente/paciente, embasada em conhecimento científico, enriquecimento do profissional e da sua prática, bem como possibilita a busca de soluções para os problemas vivenciados no cotidiano ${ }^{(16)}$.

Para a implementação da enfermagem baseada em evidências, o enfermeiro necessita ter conhecimento e competência para interpretar os resultados oriundos de pesquisa, os quais auxiliarão na tomada de decisão em relação à assistência de enfermagem. Acrescido a esse aspecto, existe a necessidade de uma cultura gerencial e organizacional que favoreça a utilização de pesquisas ${ }^{(17)}$.

A seguir, apresentaremos alguns estudos encontrados na literatura internacional, os quais abordam as barreiras para a utilização de pesquisas na enfermagem.

Em um estudo cujo objetivo consistia em analisar a atitude dos enfermeiros para com a pesquisa, os resultados evidenciaram cinco fatores considerados barreiras para a utilização de pesquisa, a saber: fator 1 barreiras pessoais (subjetivas) dos enfermeiros; fator 2 barreiras organizacionais (estruturais); fator 3 - reações dos médicos frente à pesquisa de enfermagem; fator 4 reações de profissionais da saúde e fator 5 - impacto da pesquisa de enfermagem ${ }^{(18)}$.

No fator 1 , as variáveis que obtiveram escores maiores foram: a falta de motivação, seguida pela falta de convicção do valor da pesquisa, no fator 2 , as variáveis foram a pesquisa não ser considerada como atividade do enfermeiro, e a falta de preparo e de tempo foram as que obtiveram escores maiores. Em relação ao fator 3 , as variáveis com escores maiores foram: atitude negativa da equipe médica frente à pesquisa de enfermagem seguida pela falha desses profissionais na implementação dos resultados; no fator 4 , ficou evidenciado que a falta de confiança de outros profissionais da saúde e o descrédito dos próprios enfermeiros foram as variáveis com escores mais relevantes; e, no fator 5 , a deficiência de impacto das pesquisas na prática de enfermagem foi a variável com maior escore ${ }^{(18)}$.

Em uma investigação para identificar os fatores incentivadores e desencorajadores para a utilização de pesquisas na enfermagem, as atividades de pesquisa desenvolvidas pelos enfermeiros e que obtiveram maior freqüência foram a leitura dos estudos, seguida pelo intercâmbio de informações entre os profissionais, mais do que a implementação dos resultados de pesquisas. Quando os enfermeiros foram indagados sobre o tipo de assistência necessária para incentivar a utilização dos resultados de pesquisas, esses profissionais opinaram que um método adequado e maior tempo para o desenvolvimento de atividades de pesquisa eram cruciais. Salientaram, ainda, que o auxílio de profissionais como estaticista e enfermeiro/pesquisador, bem como acesso fácil à literatura e serviços informatizados são estratégias fundamentais para a implementação dos resultados de pesquisa. A falta de tempo e de suporte organizacional foram os fatores desencorajadores que sobressaíram no estudo. Em relação aos fatores incentivadores, os enfermeiros relataram que um boletim informativo mensal sobre pesquisas é o meio mais estimulador, seguido pelos eventos científicos, programas de educação continuada, informações via computador, software e guias de estudos de pesquisas ${ }^{(19)}$.

Uma pesquisa realizada com enfermeiros perioperatórios, para identificar o interesse, barreiras e experiência com pesquisa clínica em enfermagem, evidenciou que $58 \%$ dos enfermeiros investigados relataram um interesse acurado por pesquisa, 55\% nunca tiveram aula sobre pesquisa, e apenas $40 \%$ têm lido pesquisas realizadas. Em relação às barreiras percebidas pelos participantes do estudo, a falta de tempo para realizar pesquisa, a falta de suporte organizacional e a falta de reconhecimento dos colegas foram as mais mencionadas $^{(20)}$.

Em uma investigação elaborada para identificar as barreiras para o uso da pesquisa, os sujeitos participantes apontaram como as mais significativas: tempo 
insuficiente para implementar novas idéias no trabalho, tempo insuficiente para a leitura de pesquisas, a falta da organização em adotar estratégias para facilitar a utilização de pesquisas e dificuldades na compreensão da análise estatística existente nas investigações ${ }^{(21)}$.

Os estudos mencionados até agora permitem-nos visualizar as dificuldades enfrentadas pelos enfermeiros, tanto para o desenvolvimento de pesquisas, como para a utilização de seus resultados, ou seja, mostram que a implementação da prática baseada em evidências terá que percorrer um longo caminho.

No Canadá, medidas estão em andamento para o desenvolvimento da prática baseada em evidências; hospitais e instituições de saúde têm formalmente definido o vínculo entre a pesquisa e a prática, e estratégias importantes estão sendo implementadas, como, por exemplo, a formação de comissões orientadas para a pesquisa na enfermagem, desenvolvimento de planos estratégicos, articulação de forças voltadas, para a pesquisa e o uso de modelos na prática profissional. Estão sendo introduzidos programas que identificam idéias provenientes da prática, promovem diretrizes baseadas em pesquisa e encorajem decisões baseadas na prática em evidências pela equipe de enfermagem. Os hospitais estão implementando uma variedade de medidas para promover o uso de pesquisa na prática, a saber: programas educativos para a equipe de enfermagem, desenvolvimento de projetos de pesquisa nas unidades de trabalho, clarificação do papel de pesquisador para o avanço da prática de enfermagem, por meio da utilização de pesquisas; e, em alguns serviços, o acesso à informação está sendo agilizado através da informática ${ }^{(22)}$.

Do enfermeiro perioperatório é esperado que ele seja competente tecnicamente, demonstre julgamentos independentes e tenha habilidade para a tomada de decisão. Assim, esse profissional deve ser capaz de analisar dados de pesquisa e utilizar os seus resultados para proporcionar assistência de enfermagem, com resultados positivos para o paciente, ou seja, necessita de conhecimento científico para empregar na prática profissional. Para tanto, esse profissional precisa compreender o processo de pesquisa e da investigação sistemática dos problemas da prática ${ }^{(23)}$.

Acreditamos que a implementação da prática baseada em evidências na enfermagem perioperatória poderá contribuir para a melhoria da qualidade da assistência prestada ao cliente/paciente e à redução de custos, uma vez que existem atividades desenvolvidas na prática profissional que devem ser questionadas, como, por exemplo, a degermação e escovação das mãos, o uso de proprés e o preparo da pele do paciente. Após a formulação de questões, o enfermeiro deve buscar as evidências disponíveis, ou seja, pesquisas que podem responder às questões levantadas, depois deve realizar uma avaliação dos resultados oriundos dessas pesquisas sobre a viabilidade de aplicação na prática e implementar as mudanças necessárias baseadas nas informações coletadas $^{(24)}$.

Para a implementação da prática baseada em evidências, o enfermeiro perioperatório necessita de um ambiente organizacional que proporcione recursos, para a obtenção das evidências disponíveis, e tempo, para as discussões necessárias entre os profissionais envolvidos na assistência sobre a viabilidade das mudanças necessárias. Assim, o enfermeiro, utilizando sua competência clínica e as evidências disponíveis, realizará o planejamento e implementação da assistência de enfermagem embasada em conhecimento científico ${ }^{(24)}$.

Participação em pesquisas, leitura e análise das pesquisas publicadas, aplicação dos resultados de pesquisa na prática profissional, condução de projeto de pesquisa são caminhos que o enfermeiro perioperatório deverá usar para tornar-se preparado enquanto pesquisador e melhorar suas habilidades para a tomada de decisão ${ }^{(23)}$.

\section{CONSIDERAÇÕES FINAIS}

A prática baseada em evidências vem sendo discutida na literatura e, conforme já mencionamos, várias são as barreiras para sua implementação. Entretanto, essa abordagem poderá contribuir para a mudança da prática de enfermagem baseada em tradição, rituais e tarefas para uma prática reflexiva baseada em conhecimento científico, promovendo a melhoria da qualidade da assistência de enfermagem $^{(22)}$.

O planejamento da assistência de enfermagem no período perioperatório proporcionará uma assistência integral e individualizada para o paciente cirúrgico e sua família, possibilitando a implementação de intervenções que atendam às reais necessidades do paciente, minimizando sua ansiedade e os riscos inerentes ao 
procedimento anestésico cirúrgico.

Nesse cenário, entendemos que a prática baseada em evidências é uma abordagem que incentiva o enfermeiro

\section{REFERÊNCIAS BIBLIOGRÁFICAS}

1. Jouclas VMG. Análise da função do circulante de sala de operações de acordo com a metodologia sistêmica de organização de recursos humanos. [tese]. São Paulo (SP): Escola de Enfermagem/USP; 1987.

2. Ladden CS. Conceitos básicos de enfermagem perioperatória. In: Meeker MH, Rothrock JC. Cuidados de enfermagem ao paciente cirúrgico. $10^{\mathrm{a}}$ ed. Rio de Janeiro (RJ): Guanabara Koogan; 1997. p.3-17.

3. Castellanos BEP, Jouclas VMG. Assistência de enfermagem perioperatória: um modelo conceitual. Rev Esc Enfermagem USP 1990 dezembro; 24(3):359-70.

4. Alfaro R. Application of nursing process: a step by step guide. Philadelphia: JB. Lippincott; 1986.

5. Hamer S. Evidence-based practice. In: Hamer S, Collinson G. Achieving evidence-based practice: a handbook for practitioners. London: Baillière Tindall; 1999. p.3-12.

6. Madigan EA. Evidence-based practice in home healthcare: a springboard for discussion. Home Healthcare Nurse 1998; 16(6):411-5

7. Gerrish K, Clayton J. Improving clinical effectiveness through and evidence-based approach: meeting the challenge for nursing in the United Kingdom. Nurs Adm Q 1998 summer; 22(4):55-65.

8. Sackett DL, Rosenberg WMC, Muir Gray JA, Haynes RB, Richardson WS. Evidence based medicine: what it is and what it isn't. Br Med J 1996 January; 312(7023):71-2.

9. Drummond JP, Silva E. Medicina baseada em evidências: novo paradigma assistencial e pedagógico. São Paulo (SP): Atheneu; 1998.

10. Ingersoll GL. Evidence-based nursing. Nurs Outlook 2000 July/August; 48(4):151-2.

11. Simon JM. Evidence-based practice in nursing Nurs Diag 1999 January-March; 10(1):3.

12. Stetler CB, Brunell M, Giuliano KK, Morsi D, Prince L, Stokes $\mathrm{VN}$. Evidence-based practice and the role of nursing leadership. JONA 1998 July-August; 28(7/8):45-53.

13. Muir Gray JA. Evidence based healthcare: how to make health policy and management decision. Edinburgh: Churchill Livingstone; 1997.

14. Closs SJ, Cheater FM. Evidence for nursing practice: a clarification of the issues. J Adv Nurs 1999; 30(1):10-7.

15. Hicks C, Hennessy D. Mixed messages in nursing research: their contribution to the persisting hiatus between evidence and practice. J Adv Nurs 1997; 25(3):595-601.

16. Cassiani SHB Buscando significado para o trabalho: o aperfeiçoamento profissional sob a perspectiva de enfermeiras. [tese]. Ribeirão Preto (SP): Escola de Enfermagem de Ribeirão Preto/USP; 1994.

17. Mcsherry R, Proctor-childs T. Promoting evidence-based practice through an integrate model of care: a patient case studies as a teaching method. Nurse Education in Practice 2001 March; 1(1):19-26.

18. Hicks C. A study of nurse's attitudes towards research: a factor analytic approach. J Adv Nurs 1996; 23(2):373-9. a buscar conhecimento científico por meio do desenvolvimento de pesquisas ou aplicação na sua prática profissional dos resultados encontrados na literatura.

19. Pettengill MM, Gillies DA, Clark CC. Factors encouraging and discouraging the use of nursing research findings. IMAGE: Journal of Nursing Scholarship. 1994 summer; 26(2):143-7. 20. Fawcett DL, Lainof CA. A pilot study appraising the climate for perioperative nursing research. AORN J 1996 January; 63(1):205-8.

21. Retsas $A$. Barriers to using research evidence in nursing practice. J Adv Nurs 2000; 31(3):599-606.

22. Simpson B. Evidence-based nursing practice: the state of the art. Can Nurse 1996; 92(10):22-5.

23. Csokasy JA. Building perioperative nursing research teams - part 1. AORN J 1997 February; 65(2):396-401.

24. Beyea SC. Why should perioperative RNs care about evidence-based practice AORN J 2000 July; 72(1):109-11. 